\title{
Estudo macular em olhos com óleo de silicone por meio da tomografia de coerência óptica
}

\author{
Optical coherence tomography macular study on eyes filled with silicone oil
}

\author{
Otacílio de Oliveira Maia Júnior ${ }^{1}$ \\ Walter Yukihiko Takahashi ${ }^{2}$ \\ Yoshitaka Nakashima ${ }^{3}$ \\ Hélio Paulo Primiano Júnior ${ }^{4}$ \\ Beatriz Sayuri Takahashi ${ }^{5}$ \\ Aloísio Fumio Nakashima ${ }^{6}$
}

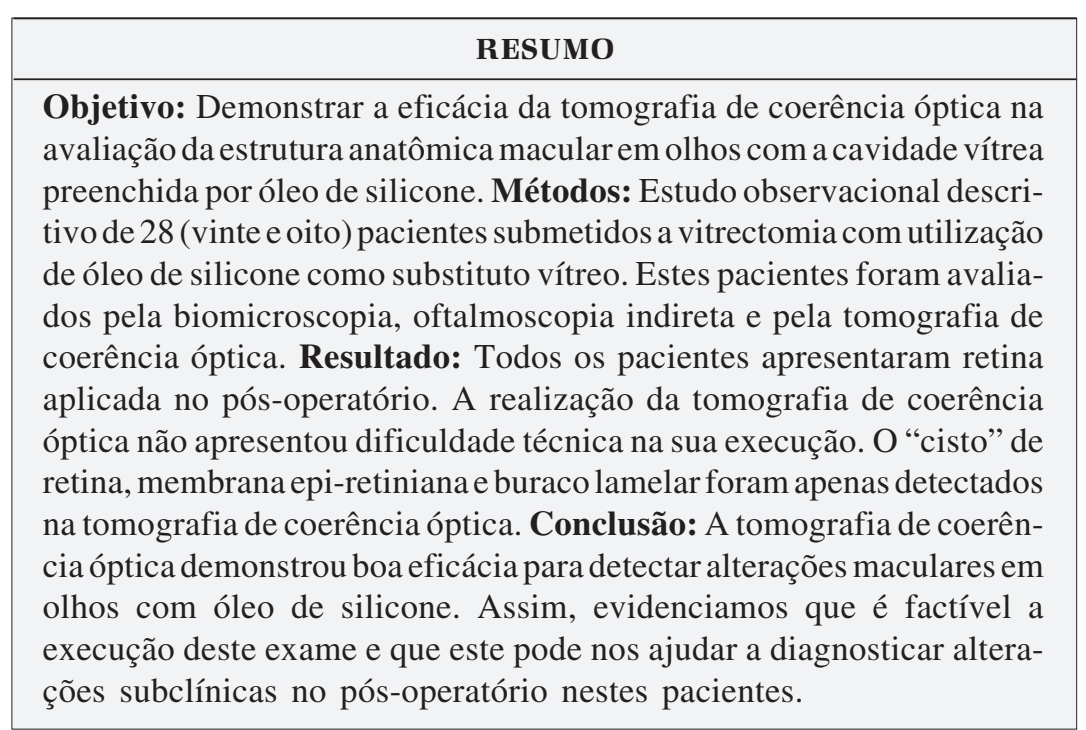

Descritores: Óleos de silicone/uso terapêutico; Vitrectomia; Tomografia de coerência óptica/métodos; Retina/cirurgia; Descolamento retiniano/cirurgia

\footnotetext{
Trabalho desenvolvido no Departamento de Oftalmologia do Hospital das Clínicas da Faculdade de Medicina da Universidade de São Paulo - USP - São Paulo (SP) - Brasil.

${ }^{1}$ Pós-Graduando (Nível Doutorado) da Faculdade de Medicina da Universidade de São Paulo e Especialista em Retina e Vítreo do Departamento de Oftalmologia da Universidade de São Paulo - USP - São Paulo (SP) Brasil.

${ }^{2}$ Médico Assistente Doutor e Chefe do Setor de Retina e Vítreo do Departamento de Oftalmologia da USP - São Paulo (SP) - Brasil.

${ }^{3}$ Médico Assistente Doutor do Setor de Retina e Vítreo do Departamento de Oftalmologia da USP - São Paulo (SP) - Brasil.

${ }^{4}$ Médico Especialista em Retina e Vítreo do Departamento de Oftalmologia da USP - São Paulo (SP) - Brasil.

${ }_{5}^{5}$ Médica Especialista em Retina e Vítreo do Departamento de Oftalmologia da USP - São Paulo (SP) - Brasil.

${ }^{6}$ Graduando da Faculdade de Medicina da USP - São

Paulo (SP) - Brasil.

Os autores declaram não possuir nenhum interesse financeiro neste estudo.

Endereço para correspondência: Otacílio de Oliveira Maia Júnior. Rua Machado Bittencourt, 29 - Apto. 53 São Paulo (SP) CEP 04044-000

E-mail: omaiausp@uol.com.br

Recebido para publicação em 25.04.2006

Última versão recebida em 27.07.2006

Aprovação em 02.08.2006
}

\section{INTRODUC̄̃̃O}

A tomografia de coerência óptica, também conhecida pela sigla em inglês OCT, é um método diagnóstico que permite fornecer imagens de alta resolução das estruturas retinianas seccionadas transversalmente, sendo uma tecnologia não invasiva e sem necessidade de contato para sua realização ${ }^{(1)}$. $O$ Stratus OCT, modelo disponível comercialmente, possui uma resolução de aproximadamente 8 a $10 \mu \mathrm{m}^{(2-3)}$. O princípio do OCT é baseado na interferometria de baixa coerência ${ }^{(4)}$ e as alterações são observadas como diferença relativa de refletividade na interface óptica das estruturas examinadas ${ }^{(1,3)}$.

Existe uma boa correlação entre imagens obtidas pelo OCT e a estrutura anatômica correspondente. Isto se deve pela diferença de refletividade das estruturas retinianas, que são representadas por uma escala de cores fornecidas pelo computador, na qual as cores brilhantes (vermelha e branca) representam alta refletividade e as escuras (azul e preta), baixa refletividade ${ }^{(5-6)}$.

O OCT pode sofrer limitações por opacidades dos meios ópticos como edema corneano, catarata ou anormalidades no humor vítreo ${ }^{(7-8)}$. O óleo de silicone é utilizado como substituto vítreo na cirurgia vítreo-retiniana para algumas situações de descolamento de retina, para manter-se aplicada até a formação de uma adesão forte de retinopexia (laserterapia) ${ }^{(9-10)}$. A presença de óleo de silicone na cavidade vítrea pode aparentemente afetar a refletividade mensurada pelo OCT e alterar a qualidade dos "scans", diminuindo sua precisão ${ }^{(11)}$. 
O objetivo deste trabalho é demonstrar a eficácia do Stratus OCT em avaliar a estrutura macular de pacientes submetidos à cirurgia vítreo-retiniana e tendo óleo de silicone como agente temporário da retinopexia.

\section{MÉTODOS}

Realizado um estudo observacional descritivo, no qual foram incluídos pacientes submetidos à cirurgia vítreo-retiniana e uso de óleo de silicone de 5000 cps do Departamento de Oftalmologia do Hospital das Clínicas da Faculdade de Medicina da Universidade de São Paulo (HC-FMUSP).

Os exames realizados no pós-operatório foram a biomicroscopia da mácula, oftalmoscopia indireta e OCT "Stratus version 4.0 software" (Carl Zeiss Meditec). As imagens obtidas pelo OCT foram realizadas no programa de medida da espessura da retina ("retinal thickness") e o cursor foi colocado manualmente no centro da fóvea, quando a depressão foveal era visível ou na parte mais espessa no centro da mácula.

As variáveis quantitativas foram expressas em médias e desvios-padrão; as qualitativas calcularam-se freqüências absolutas e relativas. O estudo foi aprovado pela Comissão de Ética para Análise de Projetos e Pesquisa da Diretoria Clínica do HC-FMUSP.

\section{RESULTADOS}

Totalizaram 28 (vinte e oito) olhos de 28 pacientes, $75 \%$ dos pacientes foram do sexo masculino e $25 \%$ feminino. A média de idade foi de 41,3 $\pm 10,7$ anos. Todos os pacientes tiveram a retina reaplicada após o tratamento cirúrgico. Em $82,1 \%$ dos casos, o OCT foi realizado no primeiro mês após a cirurgia, sendo que 71,4\% foram na segunda semana pósoperatória. Foram selecionados 7 (sete) pacientes para ilustração, pois 14 casos possuem história clínica e resultado similar ao do caso 1, 3 pacientes ao do caso 3, 2 ao descrito no caso clínico 6 e 2 ao do 7 .

\section{Caso 1}

JVS, sexo masculino, 40 anos, alto míope, no $30^{\circ}$ dia de pósoperatório (DPO) de vitrectomia e implante de óleo de silicone por descolamento de retina (DR) total, com rotura posterior e proliferação vítreo-retiniana (PVR). Apresentou, no pós-operatório, mácula aplicada com rarefação difusa do epitélio pigmentado da retina (EPR). O OCT evidenciou as camadas retinianas compatíveis com a normalidade e uma espessura macular de $162 \mu \mathrm{m}$ na região foveal (Figura 1).

\section{Caso 2}

SAE, sexo masculino, 33 anos, portador de coroideremia e retinosquise, submetido a vitrectomia e implante de óleo de silicone por DR secundário a rotura do folheto externo da retinosquise. Observou-se no pré-operatório, ao OCT, a sepa- ração das camadas intra-retinianas e o descolamento da retina neurosensorial do EPR (Figura 2A). No 20ํㅡㄹ, o OCT evidenciava uma retina aplicada, já apresentando a depressão foveal (Figura 2B).

\section{Caso 3}

OAS, sexo masculino, 25 anos, portador de uveíte posterior por toxoplasmose tratada há aproximadamente um ano, evoluindo com PVR e DR total. Foi submetido à vitrectomia, sendo utilizado perfluorcarbono para aplicação retiniana, endolaser para realização de retinopexia e óleo de silicone. No $18^{\circ} \mathrm{DPO}$ apresentava retina aplicada e as roturas bloqueadas pelas marcas do endolaser. O OCT demonstrou uma estrutura retiniana compatível com a normalidade e, anterior à depressão foveal, uma estrutura com refletividade aumentada, sendo atribuída a uma gotícula de perfluorcarbono residual (Figura 3).

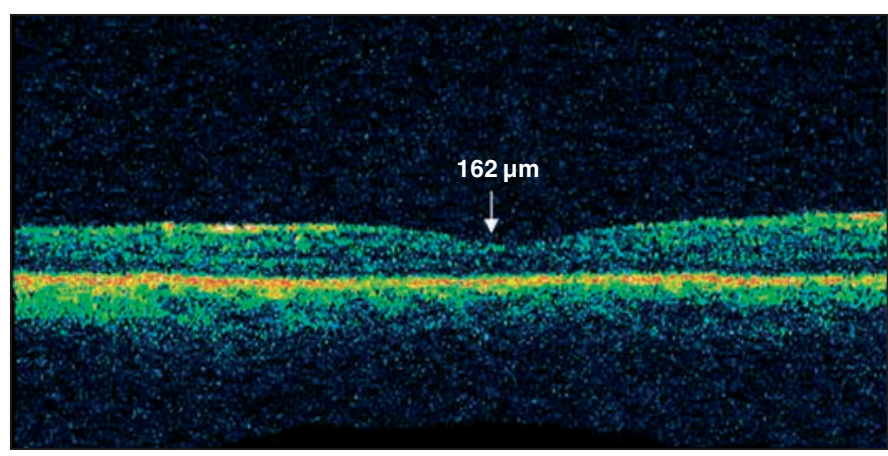

Figura $1-30^{\circ}$ dia de pós-operatório de vitrectomia com implante de óleo de silicone por descolamento total de retina
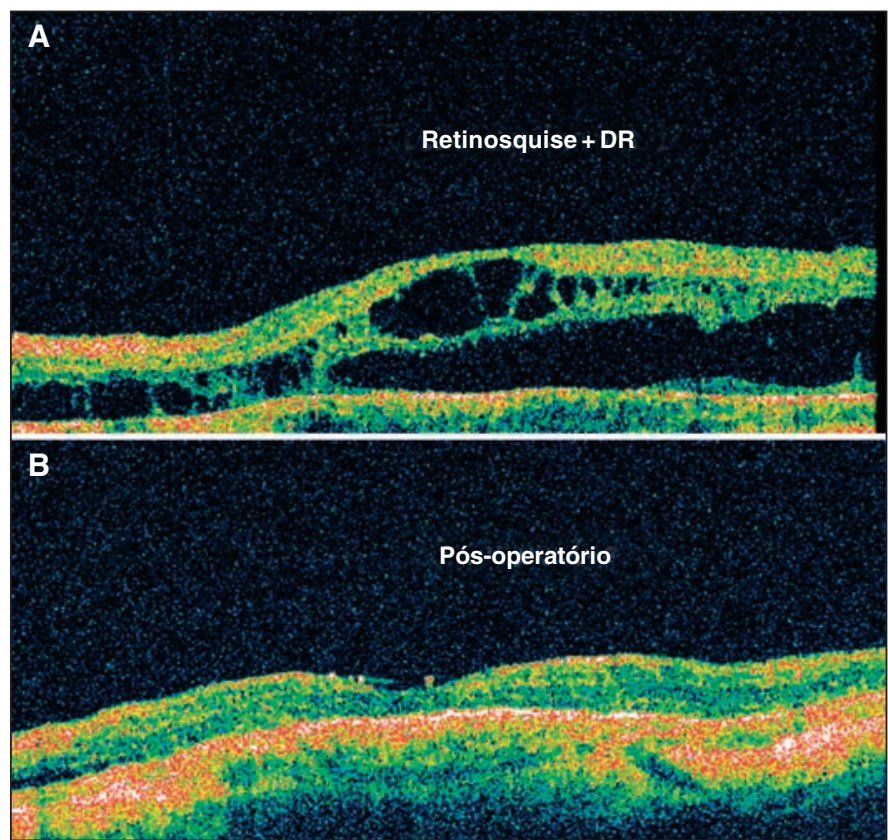

Figura 2 - A: Descolamento de retina e retinosquise num paciente com coroideremia. $\mathrm{B}: 20^{\circ}$ dia de pós-operatório de vitrectomia com implante de óleo de silicone. 


\section{Caso 4}

MLA, sexo masculino, 66 anos, portador de olho único, apresentou DR regmatogênico com mácula descolada. Foi submetido a tratamento cirúrgico com vitrectomia e implante de óleo de silicone. No $15^{\circ} \mathrm{DPO}$, a retina mostrava-se aplicada, sem alterações maculares. O OCT evidenciou uma retina aplicada e um "cisto" perifoveal de $215 \mu \mathrm{m}$ (Figura 4).

\section{Caso 5}

IIB, sexo masculino, 34 anos, vítima de trauma perfurante e corpo estranho intra-ocular (CEIO) no olho esquerdo há 8 meses, submetido à facectomia com implante de lente intraocular, vitrectomia e óleo de silicone, apresentando acuidade visual de 20/200. O exame biomicroscópico, prejudicado pela opacidade de cápsula posterior, evidenciou um edema de mácula. O OCT, além de demonstrar o espessamento macular (601 $\mu \mathrm{m}$ na região foveal), constatou uma estrutura de hiperrefletividade, anterior à membrana limitante interna, tendo como diagnóstico uma membrana epi-retiniana (MER) (Figura 5). Assim, foi programada uma intervenção cirúrgica para retirar o óleo de silicone, remover da MER e realizar uma injeção de triancinolona intravítrea para regressão do edema de macular.

\section{Caso 6}

ETA, sexo feminino, 46 anos, com baixa de acuidade visual progressiva há 10 meses e piora súbita há 5 dias. Apresentando DR total, foi submetida a vitrectomia com implante de óleo

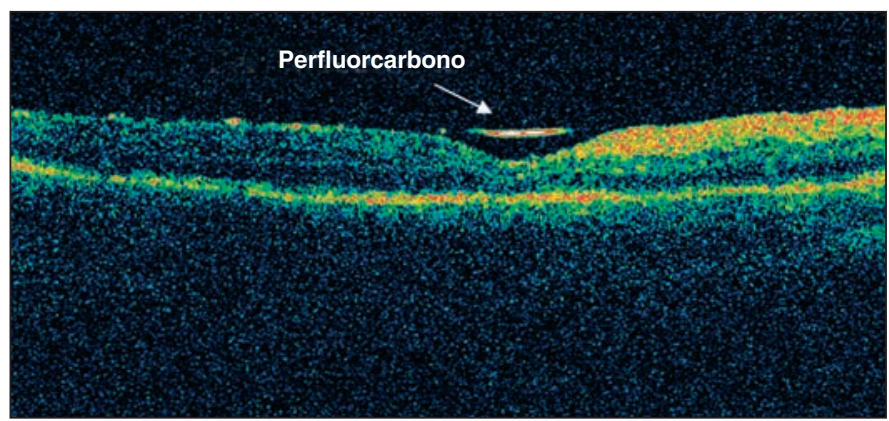

Figura 3 - Presença de perfluorcarbono residual (seta) no $18^{\circ}$ dia de pós-operatório de vitrectomia com implante de óleo de silicone

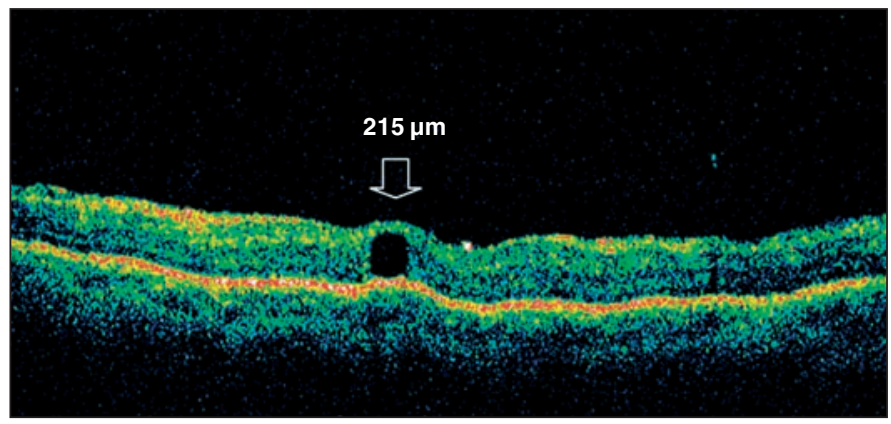

Figura 4 - Cisto intra-retiniano no $15^{\circ}$ dia de pós-operatório de vitrectomia com implante de óleo de silicone de silicone. No intra-operatório, foi visualizado um buraco macular. No $5^{\circ} \mathrm{DPO}$, a suspeita durante a cirurgia foi confirmada na biomicroscopia. Entretanto, o OCT ainda evidenciou tecido retiniano na lesão, firmando diagnóstico de buraco lamelar (Figura 6).

\section{Caso 7}

RFM, sexo feminino, 56 anos, referindo baixa de AV de longa data no olho esquerdo que "sumiu" há 1 mês. Diagnosticado DR total, foi realizada vitrectomia com implante de óleo de silicone. No $20^{\circ} \mathrm{DPO}$ foi visualizado um buraco macular. O OCT confirmou o exame clínico, demonstrando ausência completa de tecido retiniano na lesão (Figura 7).

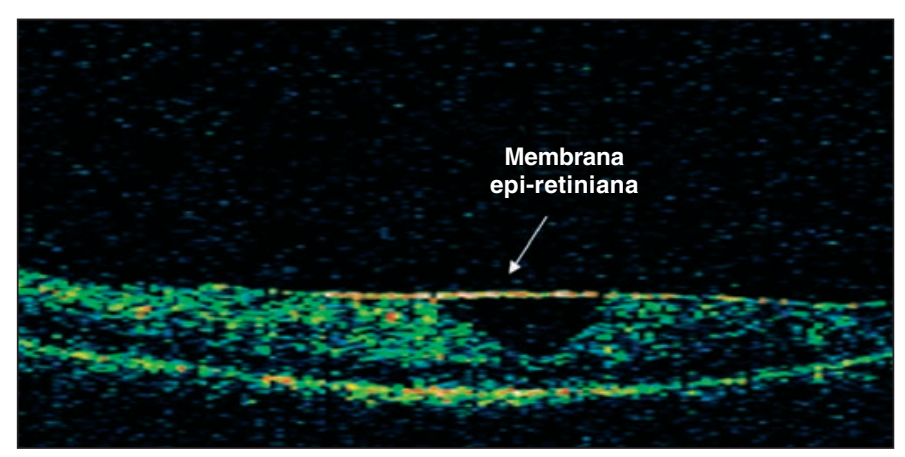

Figura 5 - Membrana epi-retiniana (seta) no pós-operatório tardio de vitrectomia com implante de óleo de silicone

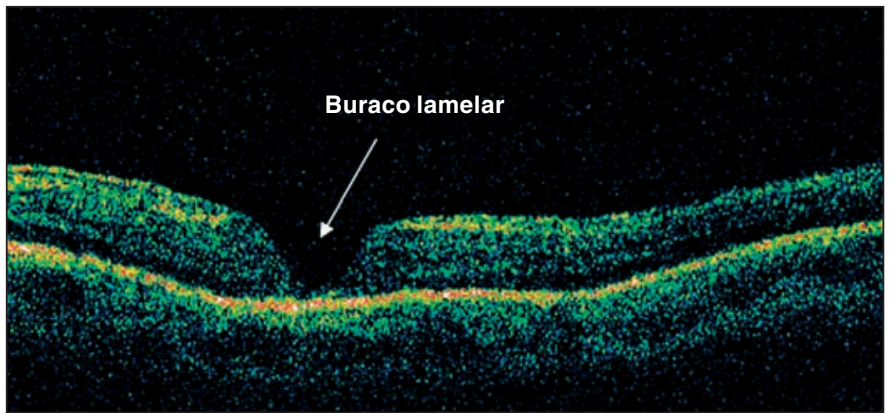

Figura 6 - Buraco lamelar no $5^{\circ}$ dia de pós-operatório de vitrectomia com implante de óleo de silicone

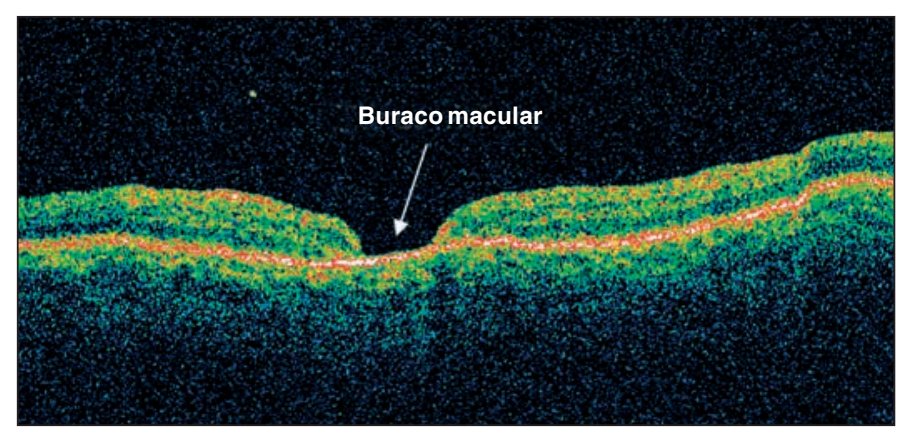

Figura 7 - Buraco macular (Grau IV) no pós-operatório de vitrectomia com implante de óleo de silicone 


\section{DISCUSS ÃO}

O mecanismo de funcionamento do OCT é similar ao da ultrasonografia, diferindo que o OCT utiliza os reflexos de ondas luminosas infravermelhas ao invés de ondas acústicas ${ }^{(12)}$. A existência de óleo de silicone representa um obstáculo na interpretação dos achados e na obtenção de imagens adequadas na ecografia $\mathrm{B}^{(13-14)}$. O silicone intra-ocular causa um aumento aparente do diâmetro axial do olho, devido à menor velocidade do som neste meio $(\text { normal }=1584 \mathrm{~m} / \mathrm{s} \text {, silicone }=980 \mathrm{~m} / \mathrm{s})^{(13,15)}$. Kumar et al., estudaram a influência da mudança de decúbito no resultado da ecografia em olhos com óleo de silicone, podendo facilitar os resultados ${ }^{(15)}$. Apesar disto, estas imagens podem ser enganadoras e requerem experiência na interpretação ${ }^{(16)}$.

Neste estudo, observou-se que as imagens obtidas pelo OCT, no pós-operatório de pacientes submetidos à cirurgia vítreo-retiniana e óleo de silicone como tamponante temporário, foram de boa qualidade, não apresentando dificuldades técnicas para execução e/ou interpretação dos resultados. As alterações refrativas induzidas pelo óleo de silicone intraocular são compensadas pelo OCT no momento de focar a estrutura retiniana, através de uma regulagem no monitor do aparelho. Esta hipermetropização é devida, fundamentalmente, ao maior índice de refração do silicone; variando, em média, de 4 a 7 dioptrias positivas ${ }^{(17-18)}$.

Alguns autores mostraram um estudo com 14 pacientes submetidos a vitrectomia e óleo de silicone (como alternativa substituta para o gás) na cirurgia de buraco macular, sendo que o seguimento desde o $1^{\circ}$ DPO foi realizado com OCT, tendo este um importante papel para detectar o fechamento do buraco, confirmando precocemente o sucesso cirúrgico ${ }^{(19)}$. Neste estudo, observou-se na oftalmoscopia indireta a aplicação retiniana em todos os casos no pós-operatório. Na biomicroscopia de fundo foram constatados edema de mácula, gotícula residual de perfluorcarbono e buraco macular não vistos na oftalmoscopia indireta. O OCT, além de demonstrar o que a biomicroscopia de fundo e a oftalmoscopia indireta evidenciaram, também constatou a presença de um "cisto" intra-retiniano, membrana epi-retiniana e buraco lamelar, não detectados pelos outros métodos propedêuticos.

\section{CONCLUSÃO}

O Stratus OCT demonstrou uma boa eficácia para detectar alterações maculares em olhos com óleo de silicone, podendo ser útil em avaliações pós-operatórias. É um exame não invasivo, de fácil execução e interpretação, podendo ser incorporado ao arsenal propedêutico no seguimento das cirurgias vítreo-retinianas, mesmo com este tamponante temporário de retinopexia.

\section{ABSTRACT}

Purpose: To demonstrate optical coherence tomography efficacy to evaluate macular anatomical outcomes, in eyes with silicone oil-filled vitreous cavity after vitrectomy. Methods: A descriptive observational study of 28 (twenty-eight) patients submitted to pars plana vitrectomy having silicone oil as vitreous substitute. The macular findings were observed by means of indirect binocular ophthalmoscope, biomicroscopy and optical coherence tomography examinations. Results: During the follow-up period the retina remained attached in all patients. In some cases, intraretinal cysts, epiretinal membrane and lamellar macular hole were observed only by optical coherence tomography examination. Conclusion: Optical coherence tomography provided improved imaging of finer retinal structures in eyes with silicone oil-filled vitreous cavity. Therefore, optical coherence tomography examination should be systematically performed in eyes filled with silicone oil to recognize changes in retinal morphology.

Keywords: Silicone oil/therapeutic use; Vitrectomy; Tomography, optical coherence/methods; Retina/surgery; Retinal detachement/surgery

\section{REFERÊNCIAS}

1. Huang D, Swanson EA, Lin CP, Schuman JS, Stinson WG, Chang W, et al. Optical coherence tomography. Science. 1991 22;254(5035):1178-81.

2. Jaffe GJ, Caprioli J. Optical coherence tomography to detect and manage retinal disease and glaucoma. Am J Ophthalmol. 2004;137(1):156-69.

3. Hee MR, Puliafito CA, Wong C, Reichel E, Duker JS, Schuman JS, et al. Optical coeherence tomography of the human retina. Arch Ophthalmol. 1995; 113(3):325-32.

4. Fercher AF, Mengedoht K, Werner W. Eye-length measurement by interferometry with partially coherence light. Opt Lett. 1988;13:186-8.

5. Puliafito CA, Hee MR, Lin CP, Reichel E, Schuman JS, Duker JS, et al. Imaging of macular diseases with optical coherence tomography. Ophthalmology. 1995;102(2):217-29.

6. Toth CA, Narayan DG, Boppart SA, Hee MR, Fujimoto JG, Birngruber R, et al. A comparison of retinal morphology viewed by optical coherence tomography and by light microscopy. Arch Ophthalmol. 1997;115(11):1425-8. Erratum in: Arch Ophthalmol 1998;116(1):77.

7. Schuman JS, Pedut-Kloizman T, Hertzmark E, Hee MR, Wilkins JR, Coker $\mathrm{JG}$, et al. Reproducibility of nerve fiber layer thickness measurements using optical coherence tomography. Ophthalmology. 1996;103(11):1889-98. Comment in: Ophthalmology. 1997;104(10):1530-1.

8. Puliafito CA, Hee MR, Schuman JS, Fujimoto JP. Interpretation of the OCT image. In: Puliafito CA, Hee MR, Schuman JS, Fujimoto JP, editors. Optical coherence tomography of ocular diseases. 2nd ed. Thorofare: Slack; 1996. p. 18-30.

9. Nehemy MB. Óleo de silicone em casos especiais de descolamento de retina. Rev Bras Oftalmol. 1988;47(6):331-7.

10. Castellarin A, Grigorian R, Bhagat N, Del Priore L, Zarbin MA. Vitrectomy with silicone oil infusion in severe diabetic retinopathy. $\mathrm{Br} \mathrm{J}$ Ophthalmol. 2003;87(3):318-21. Comment in: Br J Ophthalmol. 2003;87(10):1303-4; author reply 1304-6.

11. Mastropasqua L, Carpineto P, Ciancaglini M, Falconio G, Harris A. Reproducibility of nerve fiber layer thickness measurements using optical coherence tomography in silicone oil-filled eyes. Ophthalmologica. 2001;215(2):91-6.

12. Medeiros FA. Comparação de métodos de imagem do disco óptico e da camada de fibras nervosas da retina para o diagnóstico do glaucoma [tese]. São Paulo. Universidade de São Paulo; 2005.

13. Fisher YL. Diagnostic ophthalmic ultrasonography. In: Tasman W, Jaeger EA, editors. Duane's Foundations of clinical ophthalmology. ed rev. Philadelphia: Lippincott-Raven; 1997. p. 1-10.

14. DiBernardo C, Schachat A, Fekrat S. Ophthalmic ultrasound: a diagnostic atlas. New York: Thieme; 1998.

15. Kumar A, Sharma N, Singh R. Prone position ultrasonography in silicone filled eyes. Acta Ophthalmol Scand. 1998;76(4):496-8. 
16. Corrêa ZMS, Marcon IM. A ultra-sonografia diagnóstica (modo B) na avaliação pós-operatória das cirurgias vítreo-retinianas. Arq Bras Oftalmol. 2003;66 (1):33-8.

17. Nehemy MB, Nehemy ALG, Hilgert AHG, Garcia GFG. Alterações refrativas induzidas pelo óleo de silicone intraocular. Arq Bras Ofalmol. 1990;53(4):175-8.
18. Hotta K, Sugitani A. Refractive changes in silicone oil-filled pseudophakic eyes. Retina. 2005;25(2):167-70.

19. Jumper JM, Gallemore RP, McCuen BW 2nd, Toth CA. Features of macular hole closure in the early postoperative period using optical coherence tomography. Retina. 2000;20(3):232-7.

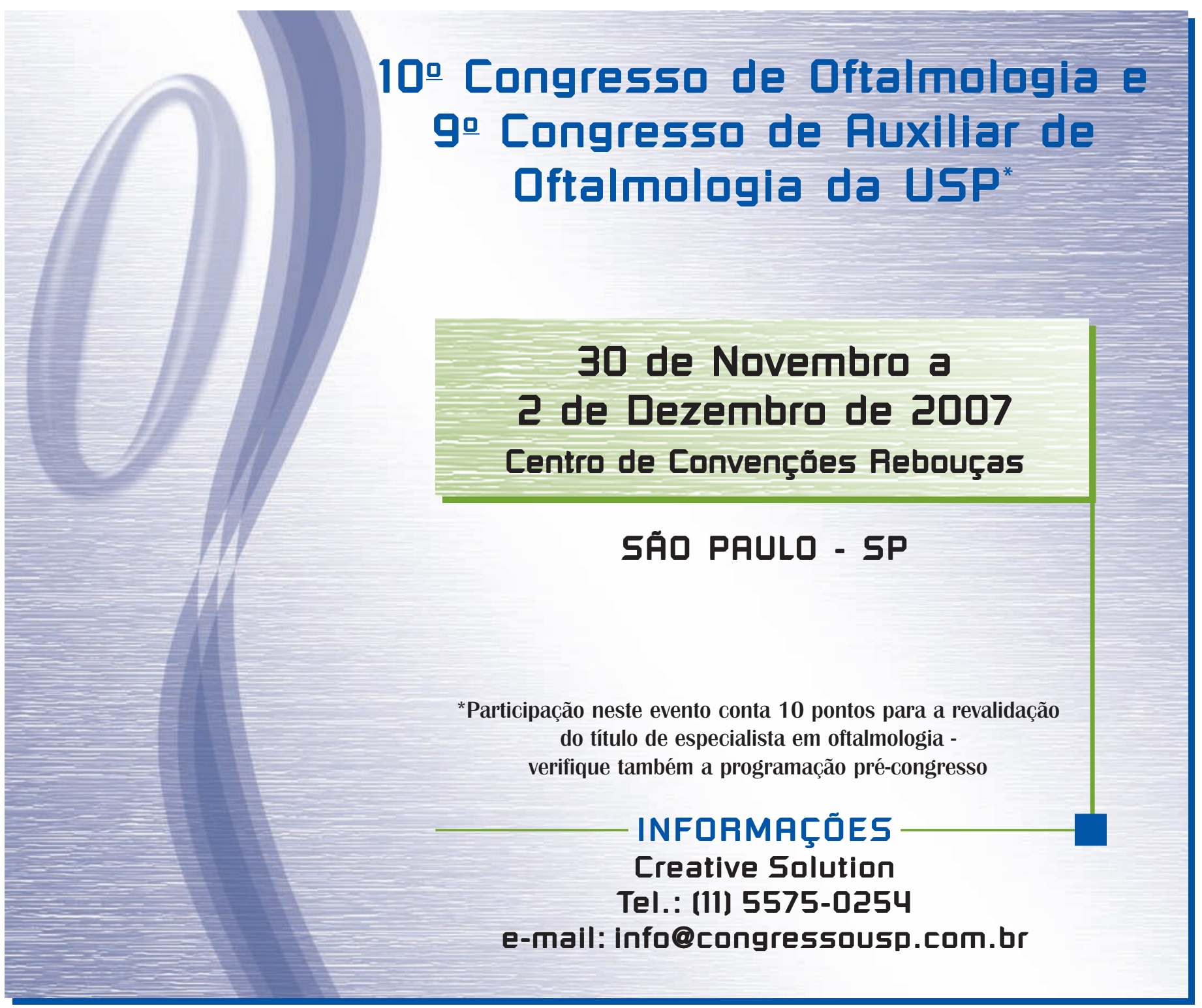

